\title{
The remarkable response to ponatinib therapy in a child with blastic phase of chronic myeloid leukemia
}

\author{
Tekin Aksu ${ }^{\oplus}$, Şule Ünal ${ }^{\oplus}$, Fatma Gümrük ${ }^{\oplus}$ \\ Division of Pediatric Hematology, Department of Pediatrics, Hacettepe University Faculty of Medicine Ankara, Turkey.
}

\begin{abstract}
Background. Chronic myeloid leukemia (CML) rarely occurs in children and adolescents, which shows more aggressive features like high risk of more advanced disease at the time of diagnosis. Suboptimal response to tyrosine kinase inhibitors (TKIs), adverse events, or advanced disease may impede the treatment.
\end{abstract}

Case. Herein we present a nine-year-old chronic phase CML case. He had no major molecular response (MMR) to imatinib, which was switched to dasatinib. MMR was ensured for 24 months, yet he developed a lymphoid blastic phase under dasatinib. He obtained a remarkable response to ponatinib when administered in parallel to multiagent induction chemotherapy.

Conclusion. Ponatinib therapy is effective and promising as a bridge to hematopoietic stem cell transplantation in children. Although more studies are necessary to determine indications, dose, efficacy, and safety data.

Key words: chronic myeloid leukemia, blastic phase, ponatinib.

Chronic myeloid leukemia (CML) accounts for $2-3 \%$ of leukemias in children and adolescents, which is less common than adults, and the disease shows more aggressive features like higher white blood cell counts (WBC), larger spleen size, and a high risk of more advanced disease at the time of diagnosis in children and adolescents. ${ }^{1,2}$ Treatment with tyrosine kinase inhibitors (TKIs) has improved outcomes and changed first-line therapy from hematopoietic stem cell transplantation (HSCT) to TKIs in children. However, there is still a risk of growth disturbance due to TKI, which is a unique side effect in pediatric patients. ${ }^{3}$ The secondgeneration (2G) TKIs such as dasatinib, nilotinib, and third-generation (3G) TKI, such as ponatinib, have expanded the treatment options that defers from HSCT. ${ }^{2}$ However, to date ponatinib has not been approved for children, and there is very limited data on

Tekin Aksu

tekinaksu@gmail.com

Received 3rd March 2020, revised 20th March 2020, accepted 23rd March 2020. dose, safety, and efficacy in children. Herein, we present a child with lymphoid blastic phase (BP) CML who had a remarkable response to ponatinib therapy when administered in parallel to acute lymphoblastic leukemia (ALL) induction treatment.

\section{Case Report}

A nine-year-old, previously healthy boy presented with fatigue, loss of weight, and bone pain. He was found to have splenomegaly, WBC of $200 \times 10^{9} / \mathrm{L}$, and $100 \% \mathrm{t}(9 ; 22)$ translocation by fluorescence in situ hybridization (FISH) analysis in Azerbaijan. Chronic phase CML (CML-CP) was diagnosed, and imatinib was initiated with a $300 \mathrm{mg} / \mathrm{m}^{2}$ dose daily. After a five-month follow-up in Azerbaijan, he presented to our center. At admission, he had complete cytogenetic response, except the BCR/ ABL1 transcript level was 0.19 international scale (IS) by polymerase chain reaction (PCR) assay. No matched family donors were present. During follow-up, he developed severe bone pain resistant to analgesics and severe neutropenia due to imatinib. At 12 months, he 
had no major molecular response (MMR) (BCR/ ABL1 0.29 IS), and imatinib was replaced with dasatinib (60 mg/m² per day). He achieved MMR at the 6th month of dasatinib therapy (BCR/ABL1, 0.09 IS). Nevertheless, the BCRABL1 transcript level was 0.04, 0.21, and 0.69 IS at 18, 21, and 24 months respectively, under dasatinib. Unfortunately, at 27 months under dasatinib, he presented with neutropenic fever, and a bone marrow aspirate revealed ALL with precursor B cell phenotype. The patient had no central nervous system or testicular involvement. Bone marrow FISH examination demonstrated $t(9 p ; 22 q)$ and the patient was started Berlin-Frankfurt-Münster-based (BFM) induction chemotherapy combined with ponatinib (15 mg daily, $13 \mathrm{mg} / \mathrm{m}^{2} /$ daily), as approved by Ministry of Health of Turkey and consent of family for this use without pediatric indication was obtained. Ponatinib started on day 15 of induction chemotherapy. Karyotyping at the time of progress to CML-BP lymphoid did not show additional chromosomal aberrations. The gene sequencing ABL kinase domain including threonine-to-isoleucine mutation at position 315 (T315I) showed no mutation at the time of the blast crisis. The blasts in the peripheral blood were disappeared in the first week of therapy. Bone marrow aspirate on day 15 showed 11\% blasts, and BCR/ABL1, 25.74 IS. He achieved hematological remission, and with a BCR/ABL1, 0.25 IS on day 33 of induction chemotherapy along with ponatinib. Complete molecular response (CMR) with a quantification limit of $0.0063 \%$ IS under the ponatinib and multiagent chemotherapy was achieved by the $3^{\text {rd }}$ month of treatment initiation. No occurrences of adverse events including QT prolongation, hypertension, and arterial occlusion, were observed. Additionally, the patient had not experienced hepatotoxicity or prolonged neutropenia in comparison with observed data from the BFM ALL trial. He underwent HSCT from a haploidentical mother (5/10 matched) after he gained CMR. At the last follow-up, he is alive with CMR. The consent of the parents of the patient is included in the hospital clinical documents.

\section{Discussion}

Recently, 2G-TKIs dasatinib and nilotinib were approved for children, along with imatinib, which was approved in 2003. Up-front TKI choice depends on efficacy, safety, availability, administration, cost, and restrictions of the insurance system. ${ }^{2}$ The limited data showed that target responses were met early, and deep molecular responses achieved with 2G-TKI, though at 18 months, are similar for imatinib.,4 The presented case demonstrated a suboptimal response to imatinib, and he experienced toxicity requiring TKI change. He sustained remission with dasatinib initially but progressed to the lymphoblastic leukemic phase subsequently. Mutations of the BCR/ABL1 kinase domain were excluded, and leukemic progression occurred despite good adherence to the drug. If failure to 2G-TKIs is observed, allogeneic HSCT is recommended. ${ }^{2}$ The prognosis of blast crisis of CML-CP while on TKI therapy is poorer than de-novo CML-BP. ${ }^{2,5}$ Millot et al. ${ }^{5}$ reported overall survival (OS) rate to be $74 \%$ at 60 months in 17 children for patients diagnosed in CML$\mathrm{BP}$, whereas an OS rate of $41 \%$ at 60 months in 21 children receiving imatinib for CML-CP progressed to predominant lymphoid blastic phase. Our patient presented with a blastic crisis under 2G-TKI, so we preferred ponatinib as a 3G-TKI, nilotinib therapy was ignored. Ponatinib therapy with BFM based induction chemotherapy was initiated as a bridge to HSCT.

There is limited experience with ponatinib in children, and a safe dose has not determined. ${ }^{6-9}$ Nickel et al. ${ }^{6}$ described an adolescent patient who lost response to imatinib due to T315I mutation. The patient was started on ponatinib at $45 \mathrm{mg}$ daily and achieved a CMR after four months of therapy. They reduced the dose to 15 mg daily to avoid toxicity. They concluded that ponatinib provided excellent disease control, but the patient had a decline in height velocity. ${ }^{6}$ Yamamoto et al. ${ }^{7}$ described a child with relapsed Philadelphia chromosome-positive ALL treated with ponatinib before HSCT. The patient received ponatinib monotherapy at 
$15 \mathrm{mg}\left(16.8 \mathrm{mg} / \mathrm{m}^{2}\right.$ per day) and achieved a complete remission at day 20 after the initiation of ponatinib without any severe adverse event. ${ }^{7}$ Millot et al. ${ }^{8}$ presented ponatinib experience in childhood Philadelphia positive leukemias in 14 patients, at a dose range of $15-41 \mathrm{mg} / \mathrm{m}^{2}$, median $27 \mathrm{mg} / \mathrm{m}^{2}$, and a median duration of 2.5 months. They concluded that ponatinib might be an additional treatment option for children with $\mathrm{Ph}+$ leukemias. ${ }^{8}$ Rossoff et al. ${ }^{9}$ reported 9 CML patients that were started on ponatinib with the median dose of 20 (range, 9-26) mg/ $\mathrm{m}^{2}$. They concluded that ponatinib was well tolerated, with no grade 4 or 5 adverse events. ${ }^{9}$

In the presented case, $15 \mathrm{mg} /$ daily ponatinib, and induction chemotherapy for ALL achieved a remarkable response without any severe toxicity. Remission can be expected with conventional chemotherapy. However, ponatinib ensured CMR at the 3rd month of therapy which was accepted as the "addon" benefit of ponatinib. Ponatinib therapy is effective and promising as a bridge to HSCT in children. However, the indications, dose, efficacy, and safety data are required.

\section{REFERENCES}

1. Hijiya N, Schultz KR, Metzler M, Millot F, Suttorp M. Pediatric chronic myeloid leukemia is a unique disease that requires a different approach. Blood 2016; 127: 392-399.
2. Hijiya N, Suttorp M. How I treat chronic myeloid leukemia in children and adolescents. Blood 2019; 133: 2374-2384.

3. Shima H, Tokuyama M, Tanizawa A, et al. Distinct impact of imatinib on growth at prepubertal and pubertal ages of children with chronic myeloid leukemia. J Pediatr 2011; 159: 676-681.

4. Gore L, Kearns PR, de Martino ML, et al. Dasatinib in pediatric patients with chronic myeloid leukemia in chronic phase: results from a phase II trial. J Clin Oncol 2018; 36: 1330-1338.

5. Millot F, Maledon N, Guilhot J, Güneş AM, Kalwak K, Suttorp M. Favourable outcome of de novo advanced phases of childhood chronic myeloid leukaemia. Eur J Cancer 2019; 115: 17-23.

6. Nickel RS, Daves M, Keller F. Treatment of an adolescent with chronic myeloid leukemia and the T315I mutation with ponatinib. Pediatr Blood Cancer 2015; 62: 2050-2051.

7. Yamamoto $M$, Hori T, Igarashi $K$, Shimada $\mathrm{H}$, Tsutsumi $\mathrm{H}$. Response to ponatinib before hematopoietic stem cell transplantation in a child with relapsed Philadelphia chromosome-positive acute lymphoblastic leukemia. Pediatr Int 2018; 60: 85-87.

8. Millot F, Suttorp M, de Bont E, et al. PF417 Ponatinib in childhood Philadelphia positive leukemias: the experience of the International Registry of childhood chronic myleloid leukemia (I-CML-Ped-Study). HemaSphere 2019; 3: 161-162.

9. Rossoff J, Huynh V, Rau RE, et al. Experience with ponatinib in paediatric patients with leukaemia. Br J Haematol 2020; 189: 363-368. 